\section{Folk appropriation of ECT among South Indian patients}

Sir: In response to the case report by Hosty (Psychiatric Bulletin, 1993, 17, 630), on selftreatment with electricity, we wish to report its use by a patient with obsessive compulsive disorder.

Mr A, aged 24, was admitted to the ward after an attempted overdose. He gave a three month history of obsessive thoughts characterised by a wish to stare at the "private parts of women". During his in-patient stay, he began frequenting the ECT clinic and talking to relatives of patients receiving ECT. This led to his demand that he be given ECT to get rid of his painful obsessions. Unsuccessful attempts to dissuade him led to anger and resentment. One day he was found to have in his possession a cardboard box containing batteries, wires and clips. He explained that, since we were unwilling to give him ECT, he had constructed his own ECT machine and was giving 'shocks' to his forehead to burn his obsessions. His notion of brain functioning revealed an electrical organ which was wired up to produce thoughts that were an end-product of electrical activity. The knowledge gained from the ECT clinic and aversion therapy at the behaviour therapy unit were proof to him that doctors used a similar model for treating mental illness.

Metaphors often diffuse out and reshape to fit prevailing folk notions in popular culture. In our experience of working with patients in South India, ECT is a popular and often highly desired form of treatment. Patients consider the ECT room a sacred place, symbolically associating it with a temple (photographs of deities, bells at the entrance with incense sticks and prayers that are frequently offered).

Nurses in uniform along with apron-clad doctors using stethoscopes and ophthalmoscopes, complete a picture that reveals a fascinating co-existence of secular and sacred symbols of healing. We have encountered many patients requesting 'current treatment' and this parallels a similar pattern of disappointment when doctors refuse to prescribe 'pills' or 'injections'. Apart from isolated research (Nichter, 1980) we know little about how biomedical ideas are appropriated by any culture to make sense of such notions (Jadhav, 1993).

JADHAV. S. (1993) (Editorial) Anthropology and medicine: bridging the link. British Medical Anthropology Soctety Bulletin. Summer, New series No 1, 1-2.

NiCHTER, M. (1980) The lay person's perception of medicine as perspective into the utilization of multiple therapy systems in the Indian context. Social Science and Medicine, 14B, 225-233.
SUSHRUT JADHAV, Centre for Medical Anthropology. University College London; and R. RAGURAM, National Institute of Mental Health and Neuroscience, Bangalore, India

\section{Attitudes to ECT}

Sir: Riordan, Barron \& Bowden (Psychiatric Bulletin, 1993, 17, 531-533) describe a reexamination of patients' attitudes towards and experience of electroconvulsive therapy (ECT). Such studies are important in keeping psychiatrists aware of consumer opinion and helping us to continually update and review our procedures. Perhaps consumer views ought to be routinely sought by consultants with responsibility for ECT clinics.

I would, however, take issue with their statement that ECT is "widely seen by the public as a barbaric and outmoded form of treatment". Freeman \& Cheshire (1986) reviewed 11 studies of patient and/or public attitudes to ECT and found generally positive views, but with considerable ignorance concerning the nature and purpose of the treatment. The possible influence of the misrepresentation of ECT in the media is a concern, but various authors disagree about the effect of publicity on attitudes towards ECT and the relative influence of different sources of information such as personal experience of the treatment, explanation from a doctor, and written information.

The authors conclude that there is a need for more explanation of ECT, more particularly for repeated explanations of the treatment throughout the course and following its completion for those receiving treatment and their relatives and visitors. The use of videotapes to inform patients about ECT as part of the consent process is contentious. Baxter and co-workers (1986) compared a group of patients who saw a factual ECT videotape during a consent process prior to treatment with ECT with a group who experienced the usual consent process (including written and verbal information). The group who saw the videotape were less sure that they had sufficient information on which to decide whether or not to consent to ECT than the comparison group. The authors suggested that videotapes might have a role in informing relatives rather than patients. Horgan (1990) has put forward a more innovative suggestion for destigmatising ECT and reassuring people receiving treatment, that partners or close relatives could be present throughout the procedure.

Freeman \& Cheshire (1986) concluded that there had been (at that time) insufficient investigation of the patient's ability to give informed consent to ECT. I suspect this is still true. 
BAXTER, L.R., ROY-BYRNE, P., LISTON, E.H. et al (1986) Informing patients about electroconvulsive therapy: effects of a videotape presentation. Convulsive Therapy, 2, 2529.

Freeman, C.P.L. \& Cheshire, K.E. (1986) Attitude studies on electroconvulsive therapy. Convulsive Therapy, 2, 31-42. HORGAN, D. (1990) Prejudice against ECT (letter). Australian \& New Zealand Journal of Psychiatry. 24, 157-158.

S. M. BENBOW, York House, Manchester Royal Infirmary, Manchester M13 9BX

Sir: We thank Dr Benbow for her comments. We agree that the Freeman $\&$ Cheshire study did not reveal strong antipathy towards ECT, but other studies (Selvin, 1987; Durham, 1989) discuss the controversies regarding its use. We have personal experience of strong negative opinions expressed by both professional colleagues and others. Combined with views expressed in the media, which must represent the views of a proportion of the population, we feel that we were justified in using the phrase "widely seen". We are aware of the anecdotal nature of some of this evidence.

Our suggestions regarding the use of other formats to aid explanation of the ECT process were not based on personal experience. We would suggest that further work, looking at the efficacy of these methods, would be invaluable.

DURHAM. J. (1989) Sources of public prejudice against electroconvulsive therapy. Australian and New Zealand Journal of Psychiatry, 23, 453-460.

SElvin. B.L. (1987) Electroconvulsive therapy. Anaesthestology. 67, 367-385

DENISE M. RIORDAN, Prestwich Hospital, Manchester M25; PHILIP BARRON, Northgate Hospital, Morpeth, Northumberland NE61 3BP; and MELANIE F. BOWDEN, formerly Manchester Royal Infirmary, Manchester M13

\section{Training in administration of ECT}

Sir: A similar survey to $\mathrm{Dr}$ Henderson's on ECT (Psychiatric Bulletin, 1993, 17, 154-155) was carried out among psychiatrists of East Berkshire, using an anonymous, self-completed questionnaire. Numbers involved were small but may be of some interest as they compared experiences of present trainees with more senior colleagues.

Questionnaires were sent to all psychiatrists in East Berkshire: seven 'trainees' $(t)$ and 13 'others' (o) (consultants, associate specialists, clinical assistants). All trainees and eight others replied. Average time in training was 103 weeks for trainees and 19 years for others. Thus most others received their training before Pillard \& Ellam's original survey of 1981 . Questions were asked about points considered to be good practice in training to administer ECT. Respondents who were no longer trainees were asked to reply with respect to their original training.

Initial questions concerned consultant cover of the ECT department. Three (t) and four (o) knew that there was a consultant responsible for training juniors. No trainees and two others recalled seeing the nominated consultant in the department regularly. Three ( $t$ ) but all others knew of an allocated person with whom they could discuss problems concerning ECT.

Two (t) and three (o) had received an initial seminar prior to administering ECT. Three $(t)$ and three (o) had the nominated consultant or his deputy present during the initial ECT session, while five (t) and five (o) had ECT demonstrated by a more senior trainee. The number of sessions observed before administering ECT alone varied. Four ( $t$ ) and three (o) had observed one session. while one of each group had observed more than one.

The study was carried out as part of an audit of ECT in our department. Concern was voiced that trainees' perception of training did not match that of the senior staff who felt that there was a consultant in charge of ECT who made a point of training juniors. The other findings were not challenged. These findings suggest that over the last 10-15 years there has been no great change in the level of training received. In most areas the 'others' did as well as or better than the 'trainees'.

PIPPARD, J. \& ELLAM, L. (1981) Electroconvulstue Treatment in Great Brtain. 1980. London: Gaskell (Royal College of Psychiatrists).

Elizabeth A. QuinN, Chilton Clinic, Warneford Hospital, Oxford OXC3 7JX

\section{Care programme approach in Shropshire district}

Sir: We would like to report our findings from a study undertaken 16 months following the inception of care programme approach (CPA) in Shropshire district. Professionals involved in the initiation and present workings of the CPA were interviewed.

The Planning and Information Department's role to monitor information, and thereby look for needs and changes for future service requirements, were not wholly met. Computer listing of allocated patients was not sent to the relevant keyworkers. This lead to lack of vital communication. The sheer volume of forms $(20,000$ a year) was too great to process with available resources and was regarded as yet another exercise in bureaucracy and red tape unlikely to effect change in clinical practice.

The community psychiatric nursing services (CPNS) were aware of their obligation to the CPA and felt it was a realistic objective despite 